\title{
Angular dependence of the magnetization of isotropic superconductors: which is the vortex direction?
}

\author{
S. Candia and L. Civale \\ Comisión Nacional de Energía Atómica, Centro Atómico Bariloche and Instituto Balseiro \\ 8400 Bariloche, RN, Argentina
}

\begin{abstract}
We present studies of the dc magnetization of thin platelike samples of the isotropic type II superconductor $\mathrm{PbTl}(10 \%)$, as a function of the angle between the normal to the sample and the applied magnetic field $\mathbf{H}$. We determine the magnetization vector $\mathbf{M}$ by measuring the components both parallel and normal to $\mathbf{H}$ in a SQUID magnetometer, and we further decompose it in its reversible and irreversible contributions. The behavior of the reversible magnetization is well understood in terms of minimization of the free energy taking into account geometrical effects. In the mixed state at low fields, the dominant effect is the line energy gained by shortening the vortices, thus the flux lines are almost normal to the sample surface. Due to the geometrical constrain, the irreversible magnetization $\mathbf{M}_{i r r}$ remains locked to the sample normal over a wide range of fields and orientations, as already known. We show that in order to undestand the angle and field dependence of the modulus of $\mathbf{M}_{i r r}$, which is a measure of the vortex pinning, and to correctly extract the field dependent critical current density, the knowledge of the modulus and orientation of the induction field $\mathbf{B}$ is required.
\end{abstract}




\section{INTRODUCTION}

It is well known that sample shape effects have a strong influence in the magnetic response of superconductors. The discovery of the high temperature superconductors (HTSC) revived the interest in these effects, particularly for the case of platelike geometries [1] because those materials are frequently produced in the form of thin films or platelike single crystals.

When we consider the shape effects on the dc magnetization of samples of type II superconductors whose dimensions are large as compared to the superconducting penetration length $\lambda$, it is useful to divide the problem in two parts, namely the reversible magnetization and the irreversible magnetization that arises from vortex pinning. The first case appears as a rather simple textbook problem (at least for geometries that can be approximated by an ellipsoid) that can be solved by using the demagnetizing tensor formalism [8,9]. The second aspect is more complex, and have received considerable recent attention, both theoretically and experimentally $[1-7]$.

The macroscopic magnetic response due to flux pinning is well described by the critical state model [10,11], according to which the current density flowing in any part of the sample is either the critical current density $J_{c}$ or zero. This current produces an spatially inhomogeneous magnetic induction $\mathbf{B}(\mathbf{r})$, that is detected in magnetometry studies as the non-equilibrium part of the magnetization. In general, $J_{c}$ is a function of the temperature $T$ and the local $\mathbf{B}(\mathbf{r})$. The simplest critical state model, proposed by Bean [11], assumes that $J_{c}$ is independent of $\mathbf{B}$, thus resulting in a uniform current density when the applied magnetic field $\mathbf{H}$ is varied in a typical isothermal measurement $\mathbf{M}(\mathbf{H})$ (hysteresis loop).

Even within the simple Bean model, the calculation of $\mathbf{B}(\mathbf{r})$ in thin samples with $\mathbf{H}$ normal to the sample surface is rather complex, and has been the subject of extensive modeling and numerical analysis [1, 2, 12, The situation is further complicated when the realistic cases of field dependent and/or anisotropic $J_{c}$ (e.g., in HTSC) are considered [13. Several experimental techniques have also been used to measure the local B [14, 3]

Another aspect of the problem, that has been less explored, is the magnetic response 
when $\mathbf{H}$ is tilted with respect to the normal to the surface of thin samples. As the nonequilibrium screening currents are strongly constrained by the geometry to flow parallel to the sample surface, there is a large angular range of applied fields in which the irreversible magnetization $\mathbf{M}_{i r r}$ points almost perpendicular to the surface [6,7], and consequently $\mathbf{B}$ is not parallel to $\mathbf{H}$. This geometrical anisotropy results in some angular effects that are qualitatively similar to those observed in extremely anisotropic (quasi two-dimensional) superconductors [15 17]. The understanding of angular effects due to sample geometry are thus necessary for a proper interpretation of those studies.

The above considerations indicate that it makes sense to explore the angular behavior of thin samples of isotropic superconductors. This is the approach followed, for instance, by Hellman et al. [6], and more recently by Zhukov et al. [0]. Those studies are mostly focused on the angular dependence of the irreversible magnetization, and little attention is paid to the modulus and angle of the magnetic induction $\mathbf{B}$, that represents the actual density and direction of the vortices.

In this work we show that in order to understand the angle and field dependence of the irreversible magnetization of thin samples, and to correctly extract the field dependent $J_{c}$

using the critical state model, a complete knowledge of the vector $\mathbf{B}$ is required. We present studies of the dc magnetization of a platelike isotropic (conventional) superconductor as a function of angle with respect to $\mathbf{H}$. We measure the total magnetization vector $\mathbf{M}$ and decompose it in its reversible and irreversible parts. We demonstrate that they have different directions, and analyze the angular and field dependence of both of them. We show that the geometrical effects on the reversible response determine the vector $\mathbf{B}$, which is the key variable in the problem.

\section{EXPERIMENTAL DETAILS AND PROCEDURES}

In the present study we used samples of the isotropic superconducting alloy $P b_{0.9} T l_{0.1}$. Two samples, a circle of $3.3 \mathrm{~mm}$ in diameter and $43 \mu \mathrm{m}$ thick and a square of $2.4 \times 2.4 \mathrm{~mm}^{2}$ 
and $39 \mu \mathrm{m}$ thick were cut from the same foil. The thicknesses were determined from the samples' weight and known density $\left(\sim 11.4 \mathrm{~g} / \mathrm{cm}^{3}\right)$, and later confirmed from the magnetic measurements. Samples dimensions were chosen so that the aspect ratios are similar to that of the $\mathrm{YBa}_{2} \mathrm{Cu}_{3} \mathrm{O}_{7-\delta}$ crystals that we use. The critical temperature is $T_{c} \sim 7.3 \mathrm{~K}$. As results from both samples are very similar, only data from the square sample will be presented here.

Dc magnetization measurements were made in a Quantum Design magnetometer equipped with two SQUID detectors, each one coupled to a set of pick up coils. This allows us to record two perpendicular components of the magnetic moment, namely the longitudinal component $m_{l}$ (parallel to $\mathbf{H}$ ), and the transverse component $m_{t}$ (normal to $\mathbf{H}$ ), and thus to determine the total magnetic moment vector $\mathbf{m}$. Samples can be rotated in situ around an axis perpendicular to $\mathbf{H}$ using a home-made sample rotator device [18]. The angular resolution, given by the minimum step, is $\sim 0.5^{\circ}$, but the overall error in the absolute value of the angle is about $\sim 2^{\circ}$. The square sample was mounted with one pair of its sides parallel to the rotation axis.

The initial alignment was performed as follows [18]. Sample was first cooled in zero field below $T_{c}$ and then a small field was applied. Sample was then rotated using the home-made system until the angle formed by its normal and $\mathbf{H}$ was about $45^{\circ}$. Subsequently, the sample was rotated around the vertical axis (parallel to $\mathbf{H}$ ) and the transverse component $m_{t}$ was measured as a function of that orientation. Once the maximum in $m_{t}$ was found, indicating that $\mathbf{m}$ lies in the plane formed by $\mathbf{H}$ and the axis of the transverse coils [19], the angular position around the vertical axis was maintained fixed throughout the rest of the experiment. (The capability to rotate around the vertical axis is provided by the commercial system and is not affected by our perpendicular rotation device).

It is known that the measurement of $m_{t}$ posses some difficulties which originate in the appearance of an spurious signal due to the longitudinal component $m_{l}$, that is detected by the transverse pick-up coils. This occurs when the sample is slightly off-centered with respect to the vertical axis of the coils [19], which is frequently the case. We have completely and satisfactorily solved this problem. The solution includes the external processing of the 
original SQUID output signal using a software developed ad-hoc. All the details related with the hardware and software of the sample rotation system are presented elsewhere [18].

We performed measurements of both components of the dc magnetization, $M_{l}=m_{l} / V$ and $M_{t}=m_{t} / V$ (where $V$ is the sample volume), as a function of applied field, at fixed temperature and for fixed values of $\Theta_{H}$, the angle formed by $\mathbf{H}$ and the normal to the sample surface, $\mathbf{n}$ (see sketch in Figure 11). We repeated these isothermal hysteresis loops for different $\Theta_{H}$. After each loop was finished, the sample was rotated to the new $\Theta_{H}$, warmed up above $T_{c}$ and cooled down in zero field (ZFC) before starting the new run. In this way, the initial Meissner response was recorded for each angle.

\section{RESULTS AND DISCUSSION}

\section{A. Meissner response as a function of angle}

Figure 1 shows a typical pair of curves of $M_{t}$ and $M_{l}$ versus $H$ for the square sample, recorded at $T=4.5 \mathrm{~K}$ using the procedure described in the previous section. In this particular case $\Theta_{H} \simeq 60^{\circ}$. Both $M_{t}$ and $M_{l}$ exhibit an initial linear dependence on $H$ characteristic of the Meissner response, followed by an hysteretic behavior indicative of vortex pinning when $H$ is further increased and then decreased.

We first analyze the Meissner response using the standard demagnetizing tensor formalism [8,9]. Let's consider a homogeneous magnetic material immersed in a uniform field $\mathbf{H}$. If the shape of the sample can be approximated by an ellipsoid, then the internal field $\mathbf{H}_{i}$, the magnetization $\mathbf{M}$ and the magnetic induction $\mathbf{B}=\mathbf{H}_{i}+4 \pi \mathbf{M}$ are all uniform, and the internal field is $\mathbf{H}_{i}=\mathbf{H}+4 \pi \overline{\bar{\nu}} \mathbf{M}$, where $\overline{\bar{\nu}}$ is the demagnetizing tensor. We thus have

$$
\mathbf{B}=\mathbf{H}+4 \pi(\overline{\overline{\mathbf{1}}}-\overline{\bar{\nu}}) \mathbf{M}
$$

(Note that Eq. 1 is valid only if $\mathbf{M}$ is uniform. It cannot be used, for instance, to describe the critical state of a superconductor). 
We apply Eq. 1 to the Meissner state, where $\mathbf{B}=0$. We decompose $\mathbf{H}$ in the two directions $\mathbf{n}$ and $\mathbf{p}$, where the unit vector $\mathbf{p}$ is defined by the intersection of the sample surface and the plane formed by $\mathbf{n}$ and $\mathbf{H}$ (see sketch in Figure 1). Then $\overline{\bar{\nu}}$ becomes diagonal [16.9] and we have $H_{\|}=H \sin \Theta_{H}=-4 \pi\left(1-\nu_{\|}\right) M_{\|}$and $H_{\perp}=H \cos \Theta_{H}=-4 \pi\left(1-\nu_{\perp}\right) M_{\perp}$. For very thin samples $\nu_{\|} \approx \nu$ and $\nu_{\perp} \approx 1-2 \nu$ with $\nu \ll 1$, thus

$$
\begin{aligned}
4 \pi m_{l} & =-\left(\frac{1}{2 \nu} \cos ^{2} \Theta_{H}+\frac{1}{1-\nu} \sin ^{2} \Theta_{H}\right) V H \\
4 \pi m_{t} & =-\left(\frac{1}{2 \nu}-\frac{1}{1-\nu}\right) \sin \Theta_{H} \cos \Theta_{H} V H
\end{aligned}
$$

where we have written eqs. 2 in terms of the measured quantities $m_{l}$ and $m_{t}$. A simple way to test this dependence is to make a ZFC, then apply a small field (typically $\sim 10 O e$ ) and rotate the sample at fixed $H$. One of such measurements is shown in Figure 2, where the solid lines are fits to Eqs. 2. The small discrepancies between the data and the fits are due to the fact that the ZFC procedure is not perfect and thus there is always a small remanent field. This field induces a remanent magnetic moment in the sample that adds to the Meissner signal in Eqs. 2. The periodicity of this remanent moment is $360^{\circ}$, instead of the $180^{\circ}$ periodicity of the Meissner signal. When that effect is taken into account, we obtain excellent fits to the data in fig. 2, as described elsewhere [18].

A more exact method is to determine the longitudinal and transverse Meissner slopes $m_{l}^{\prime}=\frac{d m_{l}}{d H}$ and $m_{t}^{\prime}=\frac{d m_{t}}{d H}$ from the initial part of the magnetization loops $\mathbf{M}(\mathbf{H})$. This procedure eliminates the uncertainty in the value of $H$ due to the remanent field, and also that arising from remanent magnetic moments, as they are field independent. In the inset of figure 2 we have plotted $m_{l}^{\prime}$ and $m_{t}^{\prime}$ as a function of $\Theta_{H}$ for all the angles measured. We obtain excellent agreement with eqs. 2 with the parameters $V=2.29 \times 10^{-4} \mathrm{~cm}^{3}$ and $\nu=0.018$. The volume so obtained coincides within $1 \%$ with the volume calculated by weighting, and it is the value that we have used to calculate the curves of figure 1 and all the magnetization data shown from now on. The demagnetizing factor coincides very well with the expected value $\nu \simeq t / L=0.016$. 
It is also useful to calculate the modulus and orientation of $\mathbf{M}$ in the Meissner state from Eqs. 2. If we call $\alpha$ the angle formed by $\mathbf{M}$ and $\mathbf{H}$, then $\tan \alpha=\left(m_{t}^{\prime} / m_{l}^{\prime}\right)$ is

$$
\tan \alpha=\frac{(1-2 \nu) \tan \Theta_{H}}{1+2 \nu \tan \Theta_{H}^{2}}
$$

while the modulus is

$$
-4 \pi M=\left[\frac{\sin \Theta_{H}^{2}}{(1-\nu)^{2}}+\frac{\cos \Theta_{H}^{2}}{(2 \nu)^{2}}\right]^{1 / 2} H
$$

We found that Eqs. 2 and 3 allow us to determine the angle $\Theta_{H}$ from the initial slopes

of our $\mathbf{M}(\mathbf{H})$ data with accuracy better than $1^{\circ}$. As this error is lower than that associated with our mechanical devise $\left(\sim 2^{\circ}\right)$, we decided to use in all cases the value of $\Theta_{H}$ obtained from the Meissner slopes, and take the reading of the rotator as a double-check.

\section{B. The reversible response in the mixed state}

In this section we will show that the reversible response of the sample behaves as expected in an isotropic superconductor. Assuming that the Bean model is valid, both the longitudinal and transverse components of the equilibrium or reversible magnetization $\mathbf{M}_{e q}$ in the mixed state, $M_{e q, l}$ and $M_{e q, t}$, can be determined by simply taking the mean value between the two branches of the hysteresis loops, as illustrated in figure 1. We see that for fields above $\sim 500 O e$ the transverse component $M_{e q, t} \approx 0$. This is true for all $\Theta_{H}$, i.e., at high fields $\mathbf{M}_{e q}$ is parallel to $\mathbf{H}$ regardless of the sample orientation.

In figure 3 we show the modulus $M_{e q}$ as a function of $H$ for several field orientations. Also shown are the corresponding Meissner responses, which are angle dependent as expected from Eq. 1. On the other hand, in the mixed state at high fields (above $\sim 5000 e$ ) all the curves coincide; and in particular the condition $M_{e q} \approx 0$ that determines the upper critical field $H_{c 2}$ is independent of $\Theta_{H}$.

The behavior of $\mathbf{M}_{e q}$ in the mixed state at high fields is a consequence of the isotropy of the material. Indeed, the free energy $F$ of the vortex system in isotropic superconductors 
is only a function of $B$, and the equilibrium state is obtained by minimization of the Gibbs free energy [see Eq. 8.60 in [16]]

$$
G=F(B)-\frac{B^{2}}{8 \pi}+\frac{(\mathbf{B}-\mathbf{H}) \mathbf{M}_{e q}}{2}
$$

at high fields $4 \pi M_{e q} \ll H$, then $\mathbf{B} \approx \mathbf{H}$, and the last term in Eq. 5 is negligible. We thus recover the solution of an infinite superconductor, where $\mathbf{B}, \mathbf{H}$ and $\mathbf{M}_{e q}$ are parallel and $M_{e q}(H)$ is given by the usual expressions [8] regardless of the sample geometry.

The penetration of the first vortex becomes energetically favorable when the modulus of the internal field, $H_{i}$, equals the lower critical field $H_{c 1}$. Taking into account that in the Meissner state $\mathbf{H}=(\overline{\overline{\mathbf{1}}}-\overline{\bar{\nu}}) \mathbf{H}_{\mathbf{i}}$, we can define an "apparent" lower critical field $H_{c 1}^{*}\left(\Theta_{H}\right)$ that, in spite of the material isotropy, is angle dependent due to geometrical effects:

$$
H_{c 1}^{*}=H_{c 1}\left[\frac{\sin \Theta_{H}^{2}}{(1-\nu)^{2}}+\frac{\cos \Theta_{H}^{2}}{(2 \nu)^{2}}\right]^{-1 / 2}
$$

The experimental determination of $H_{c 1}^{*}\left(\Theta_{H}\right)$ is complicated by the fact that for fields only slightly above the Meissner regime, the critical state is not fully developed in the increasing branch of the loop (see figure [), and the mean magnetization does not give us a good estimate of $M_{e q}$. To solve this difficulty we adjusted the $M_{e q}(H)$ data at intermediate fields by a dependence $M_{e q} \propto \ln H$. Although this logarithmic dependence is only expected in high $\kappa$ superconductors [16,8], while in the present case $\kappa \sim 2.2$, the experimental fact is that the fit works pretty well. The upper limit of the field range of validity of the fit is about $H \simeq 500 O e$. The lower limit depends on $\Theta_{H}$, varying from $H \simeq 150 O e$ for $\Theta_{H} \simeq 0^{\circ}$ to $H \simeq 250 O e$ for $\Theta_{H}$ approaching $90^{\circ}$. We then identified the field at which the extrapolation of the fits intersect the Meissner slope with $H_{c 1}^{*}\left(\Theta_{H}\right)$, as shown in figure 3 .

Evidence that the extrapolations provide good estimates of $M_{e q}$ come from the observation that the intersection with the Meissner slopes occur at approximately the same magnetization value $4 \pi M_{e q} \sim 290 O e$ for all angles. This is what we would expect, as from Eqs. 因 and 6 results $4 \pi M_{e q}\left(H_{c 1}^{*}\right)=H_{c 1}$, independent of $\Theta_{H}$. 


\section{The vortex orientation}

We can now use the $\mathbf{M}_{e q}$ data to calculate the equilibrium induction field $\mathbf{B}_{e q}$ according to Eq. 1. In figure 4 we plot the angle $\Theta_{B}$ between $\mathbf{B}_{e q}$ and $\mathbf{n}$, as a function of $H$ for several orientations $\Theta_{H}$. At high fields $\Theta_{B} \approx \Theta_{H}$, as already discussed, but as $H$ decreases $\Theta_{B}$ approaches to zero for all orientations.

This behavior is also understood from thermodynamic considerations. From Eq. 5 we can obtain the equilibrium condition by minimization with respect to $\mathbf{B}_{e q}$, i.e. $\frac{\partial G}{\partial B_{e q, \perp}}=$ $\frac{\partial G}{\partial B_{e q, \|}}=0$. Combining with Eq. [1 these conditions result

$$
\begin{gathered}
\frac{\partial F}{\partial B_{e q, \perp}}-\frac{B_{e q, \perp}}{4 \pi}+\frac{\left(B_{e q, \perp}-H_{\perp}\right)}{8 \pi \nu}=0 \\
\frac{\partial F}{\partial B_{e q, \|}}-\frac{B_{e q, \|}}{4 \pi}+\frac{\left(B_{e q, \|}-H_{\|}\right)}{4 \pi(1-\nu)}=0
\end{gathered}
$$

For $\nu \ll 1$, the first expression implies that $B_{e q, \perp} \approx H_{\perp}$ and the second condition reduces to $\frac{\partial F}{\partial B_{e q, \|}} \approx \frac{H_{\|}}{4 \pi}$. For fields slightly above $H_{c 1}^{*}$, vortex density is very low $\left(B_{e q} \ll H_{c 1}\right)$, then interactions are negligible and $F \approx\left(B_{e q} / \Phi_{0}\right) \varepsilon_{l}$, where $\varepsilon_{l}$ is the vortex line energy [16]. In this limit $B_{e q, \|} / H_{\|} \approx B / H_{c 1}$, thus $B_{e q, \|} \ll H_{\|}$, i.e., the vortex direction is very close to $\mathbf{n}$. At intermediate fields $F \approx \frac{B_{e q}^{2}}{8 \pi}+\frac{\Phi_{0} B_{e q}}{2(4 \pi \lambda)^{2}} \ln \left(H_{c 2} / B_{e q}\right)$ and we obtain

$$
B_{e q, \|} \approx H_{\|}-\frac{\Phi_{0}}{8 \pi \lambda^{2}} \frac{B_{e q, \|}}{B} \ln \left(\frac{H_{c 2}}{e B_{e q}}\right)
$$

The physical interpretation of these results is straightforward. The normal component remains almost unchanged due to flux conservation (a result that of course becomes exact in the limit of an infinite slab). At low fields the parallel component is reduced because the system gains energy by shortening the vortex length. As field increases interactions also favor the reduction of $B_{e q, \|}$, but eventually the energy cost of having $\mathbf{B}_{e q}$ non-parallel to $\mathbf{H}$ becomes too large. Thus, vortex orientation shifts from $\mathbf{n}$ to $\mathbf{H}$ as field increases. The modulus $B_{e q}$ evolves from $B_{e q} \sim H_{\perp}$ at low fields to $B_{e q} \sim H$ at high fields. 


\section{The irreversible response}

According to the Bean model [11], the irreversible magnetization is related to $J_{c}$ through a geometry dependent proportionality constant. We define the irreversible magnetization vector as $\mathbf{M}_{i r r}=\frac{1}{2} \Delta \mathrm{M}$, where the components of $\Delta \mathrm{M}$ are the widths of the longitudinal and transverse hysteresis loops, $\Delta M_{l}$ and $\Delta M_{t}$ respectively. In thin samples the non-equilibrium currents are constrained to the sample plane, thus they should generate an irreversible magnetization $\mathbf{M}_{i r r}$ that is almost locked to the sample normal [7] in a wide angular regime $0 \leq \Theta_{H} \leq \Theta_{c}$, where $\tan \left(\Theta_{c}\right) \sim L / t$. When $\Theta_{H} \sim \Theta_{c}$, the direction of $\mathbf{M}_{i r r}$ is expected to rotate $\sim 90^{\circ}$ in a narrow angular range and to locate in the plane of the plate. This behavior has been recently demonstrated by Zhukov et al. [7] in detailed studies of the modulus and orientation of the irreversible magnetization of both isotropic and anisotropic thin superconductors.

We have confirmed that in our samples, for $\Theta_{H} \leq 70^{\circ}$ and for all applied fields, $\mathbf{M}_{i r r}$ points in the direction of $\mathbf{n}$ within our $1^{\circ}$ resolution. For angles $\Theta_{H} \geq 70^{\circ}$ the situation is more complex. $\mathbf{M}_{\text {irr }}$ progressively tilts away from $\mathbf{n}$ as $\Theta_{H}$ increases, but its direction is field dependent. This behavior cannot be described by the expressions proposed by Zhukov et al. [7], where the field dependence of $J_{c}$ is not considered.

In figure 5(a) we show the modulus $M_{i r r}$ as a function of $H$ for different angles $\Theta_{H}$. For clarity we only show data up to $\Theta_{H}$ slightly above $70^{\circ}$, thus all the curves in the figure correspond to $\mathbf{M}_{i r r} \| \mathbf{n}$ or very close to it. We first note that $M_{i r r}$ goes to zero for all $\Theta_{H}$ at $H=H_{c 2}$, as expected for an isotropic type-II superconductor. Another evident result is that, although our superconductor is isotropic, $M_{i r r}$ is angle dependent, even for $\Theta_{H} \leq 70^{\circ}$. We will now show that this dependence originates in sample geometry effects.

Clearly, to understand these results we must go beyond the Bean model and consider the field dependence of $J_{c}$. There are two basic points to be taken into account. The first one is that $J_{c}$ (and consequently $M_{i r r}$ ) is a function of $B$ rather than $H$. The second one is that the non-equilibrium currents determine the direction of the variation of $\mathbf{B}(\mathbf{r})$ 
(through $\mathbf{J} \propto \nabla \times \mathbf{B}$ ) but not the vortex orientation itself. Indeed, the field distribution in the critical state of thin samples, either for $\Theta_{H}=0$ or for $\Theta_{H} \neq 0$, has been calculated taking into account the contribution of the irreversible currents plus the applied field, but not the reversible magnetization [1].

We will consider that the field $\mathbf{B}(\mathbf{r})$ arises from the superposition of the spatially inhomogeneous contribution produced by the non-equilibrium currents and the homogeneous reversible contribution calculated in the previous section. Provided that the irreversible contribution to $\mathbf{B}(\mathbf{r})$ is not too large as compared to $\mathbf{B}_{e q}$, as indeed occurs in our samples, then the average vortex orientation is still approximately given by $\mathbf{B}_{e q}$. This also allows us to make the approximation that $M_{i r r}$ is simply a function of $B_{e q}$. (This is equivalent to the usual approach of considering that, although $J_{c}$ is field dependent, it is almost constant over the range of field variation within the sample for a given $H$, and thus the Bean model can be applied to each field). At low fields we can further approximate $B_{e q} \sim H_{\perp}$, thus we expect $M_{i r r}$ to be a function of $H_{\perp}$.

To check the above modeling, in figure 5(b) we re-plot the $M_{i r r}$ data of fig. 5(a), as a function of $H_{\perp}$. The good scaling of the curves in the low field range indicates that the angular dependence of $M_{i r r}$ in this isotropic superconductor can be understood in terms of the angular-dependent equilibrium vortex orientation.

In figure 6 we show $M_{i r r}$ as a function of $\Theta_{H}$ for several $H$. At very low fields $(H \leq 10 O e)$, $M_{i r r}$ is almost constant over a wide angular range, starts to decrease at around $\Theta_{H} \sim 70^{\circ}$ and reaches its minimum value at $\Theta_{H}=90^{\circ}$. This is, at least qualitatively, the expected behavior [7] in thin samples when the field dependence of $J_{c}$ is not taken into account. Indeed, the dotted line is the dependence predicted by Zhukov et al [7], where we have used the aspect ratio $\nu \sim 0.018$ of our sample determined previously, and thus there are no free parameters. We note, however, that a better fit could be obtained using a larger $\nu$.

At higher fields we must take into account the vortex orientation as described above. On the other hand, for $\Theta_{H} \leq 70^{\circ}$ we do not need to consider the reduction of $M_{i} r r$ due to the geometrical effects described in [0]. We thus expect an angular dependence 


$$
M_{i r r}\left(H, \Theta_{H}\right)=M_{i r r}\left(H_{\perp}, \Theta_{H}=0\right)=M_{i r r}^{0}\left(H \cos \Theta_{H}\right)
$$

where $M_{i r r}^{0}$ is the curve measured for $\Theta_{H}=0$. The validity of this scaling is manifested

in the figure 6 . An excellent agreement between the experimental data and eq. 8 is observed for $\Theta_{H} \leq 70^{\circ}$ in the field range $0 \leq H \leq 240 O e$, where $B$ is approximately aligned with the normal to the sample. For $\Theta_{H}>70^{\circ}$ the decrease of $M_{\text {irr }}$ due to sample geometry takes over. For fields above $240 O e$, the fit fails because the $B_{e q} \sim H_{\perp}$ approximation is not satisfied. We note that this model satisfactorily accounts for the angular dependence of $M_{i r r}$ that Zhukov et al. [7] observed at high fields in their isotropic samples, which could not be explained within their picture.

\section{CONCLUSION}

We have shown that the variable that governs the angle dependence of $M_{i r r}$ in isotropic thin samples is the magnetic induction $\mathbf{B}$, which describes the density and orientation of the vortices. The behavior of $M_{i r r}\left(H, \Theta_{H}\right)$ at low fields and over a wide angular range can be understood by simultaneously taking into account the modulus and orientation of the equilibrium magnetization vector $\mathbf{B}_{e q}$, and the field dependence of $M_{i r r}$ when $\mathbf{H}$ is normal to the sample. To perform the analysis reported here, it was necessary to simultaneously measure both components of the magnetization in an isotropic sample of well defined geometry, and to be able to decompose it in the reversible and irreversible parts. This last step was only possible because we used a sample with very low $J_{c}$.

It is a well known experimental fact [20] that HTSCs exhibit the same scaling of $M_{i r r}$ with $H_{\perp}$ as described by Eq. 8. That behavior is consistent with the anisotropic scaling expected for these materials in the limit of very large anisotropy [15.16], and is usually taken as evidence for quasi two dimensional behavior. However, we have observed that the same scaling occurs in our isotropic sample, thus indicating that geometrical effects should be carefully taken into account in those studies. 


\section{REFERENCES}

[1] Clem J R and Sanchez A 1994 Phys. Rev. B. 509355

[2] Brandt E H 1994 Phys. Rev. B. 49 9024; 1994 Phys. Rev. B. 50 4034; 1995 Phys. Rev. B. 5215442

[3] Schuster T, Kuhn H, Koblischka M R, Theuss H, Kronmüller H, Leghissa M, Kraus M and Saemann-Ischenko G 1993 Phys. Rev. B. 47 373; Schuster Th, Kuhn H, Brandt E H, Indenbom M V, Kläser M, Müller-Vogt G, Habermeier H-U, Kronmüller H and Forkl A 1995 Phys. Rev. B. 52 10375; Schuster T, Leghissa M, Koblischka M R, Kuhn H, Kraus M, Kronmüller H and Saemann-Ischenko G 1992 Physica C 203203

[4] Frankel D J 1979 J. Appl. Phys. 505402

[5] Kunchur M N and Poon S J 1991 Phys. Rev. B. 432916

[6] Hellman F, Gyorgy E M and Dynes R C 1992 Phys. Rev. Lett. 68867

[7] Zhukov A A, Perkins G K, Bugoslavsky Yu V and Caplin A D 1997 Phys. Rev. B. 56 2809

[8] Parks R D (ed.) 1969 Superconductivity Marcel Dekker Inc., New York

[9] Landau L D and Lifshitz E M 1960 Electrodynamics of Continuous Media Pergamon Press Ltd., Oxford

[10] Campbell A M and Evetts J E 1972 Adv. Phys. 21199

[11] Bean C P 1962 Phys. Rev. Lett. 8250

[12] Daeumling M and Larbalestier D C 1989 Phys. Rev. B. 409350

[13] Conner L W and Malozemoff A P 1991 Phys. Rev. B. 43402

[14] Tamegai T, Krusin-Elbaum L, Santhanam P, Brady M J, Feild C and Holtzberg F 1992 Phys. Rev. B. 452589 
[15] Blatter G, Geshkenbein V B and Larkin A I 1992 Phys. Rev. B. 6875

[16] Blatter G, Feigel'man M V, Geshkenbein V B, Larkin A I and Vinokur V M 1994 Rev. Mod. Phys. 41125

[17] Buzdin A I and Simonov A Yu 1991 Physica C 175143

[18] Casa D M et al. to be published

[19] Quantum Design operating manual and application notes

[20] Roas B, Schultz L and Saemann-Ischenko G, 1990 Phys. Rev. Lett. 64 479; Schimtt P, Kummeth P, Schulz L and Saemann-Ischenko G 1991 Phys. Rev. Lett. 67267 


\section{FIGURES}

FIG. 1. (a) Open symbols: Transverse component of the magnetization as a function of applied field for the sample normal at an angle of $\sim 60^{\circ}$. The arrows indicate the direction of the field sweep. Full symbols: Mean value of the magnetization. (b) Idem for the longitudinal component. The sketch shows the components and angles used in the text.

FIG. 2. Angular dependence of the longitudinal and transverse components of the magnetization in the Meissner state, as measured by rotating the sample at fixed applied field (the origin of angles is arbitrary). The solid lines are fits to Eq. 2. Inset: Meissner slopes as a function of angle. The solid lines are fits to Eq. 2, see text.

FIG. 3. Modulus of the equilibrium magnetization in the Meissner and mixed states as a function of applied field, for several sample orientations. The dotted lines are fits $M_{e q} \propto \ln H$. The inset shows a blow up of the same data in semilogarithmic scale. The intersections of the extrapolations of the logarithmic fits with the Meissner response (shown by crosses) provide an estimate of $H_{c 1}^{*}\left(\Theta_{H}\right)$

FIG. 4. Angle between the equilibrium induction field $\mathbf{B}$ and the normal to the sample, as a function of applied field, for three sample orientations $\Theta_{H}$. The symbols are experimental data; the dotted lines are the extrapolations of the logarithmic fits shown in figure 3 .

FIG. 5. (a): Irreversible magnetization as a function of applied field for several sample orientations. (b): Same data, as a function of the normal component of the applied field.

FIG. 6. Irreversible magnetization as a function of $\Theta_{H}$ for several applied fields. From top to bottom, $H=0,20,30,40,50,60,70,80,100,120,160,200,240 O e$. The solid lines are fits to Eq. 8. The dotted line is a fit to the model of Ref. [7] using an aspect ratio $\nu=0.018$ 